\title{
On a diophantine inequality involving prime numbers
}

by

\author{
D. I. TOlev (Plovdiv)
}

In 1952 Piatetski-Shapiro [4] considered the following analogue of the Goldbach-Waring problem.

Assume that $c>1$ is not integer and let $\varepsilon$ be a positive number. If $r$ is a sufficiently large integer (depending only on $c$ ) then the inequality

$$
\left|p_{1}^{c}+p_{2}^{c}+\ldots+p_{r}^{c}-N\right|<\varepsilon
$$

has a solution in prime numbers $p_{1}, \ldots, p_{r}$ for sufficiently large $N$. More precisely, if the least $r$ such that (1) has a solution in prime numbers for every $\varepsilon>0$ and $N>N_{0}(c, \varepsilon)$ is denoted by $H(c)$ then it is proved in [4] that

$$
\limsup _{c \rightarrow \infty} \frac{H(c)}{c \log c} \leq 4
$$

Piatetski-Shapiro also proved that if $1<c<3 / 2$ then $H(c) \leq 5$. One can conjecture that if $c$ is near to unity then $H(c) \leq 3$. On the other hand, instead of fixed $\varepsilon$, we may consider $\varepsilon$ depending on $N$ and tending to zero as $N$ tends to infinity.

This conjecture is proved in [7] for $1<c<27 / 26$ and

$$
\varepsilon=N^{-(1 / c)(27 / 26-c)} \log ^{13} N \text {. }
$$

In this paper we sharpen the last result and prove the following theorem:

Theorem. Let $1<c<15 / 14$. There exists a number $N_{0}(c)>0$ such that for each real number $N>N_{0}(c)$ the inequality

$$
\left|p_{1}^{c}+p_{2}^{c}+p_{3}^{c}-N\right|<N^{-(1 / c)(15 / 14-c)} \log ^{9} N
$$

has a solution in prime numbers $p_{1}, p_{2}, p_{3}$.

\section{Notation}

$c$ - fixed real number such that $1<c<15 / 14$,

$N$ - sufficiently large number, 


$$
\begin{aligned}
X & =(N / 2)^{1 / c}, \\
\tau & =X^{13 / 28-c / 2}, \\
T & =X^{41 / 56+c / 4}, \\
\varepsilon & =X^{-(15 / 14-c)} \log ^{8} X, \\
\Delta & =\frac{1}{10} \varepsilon, \\
r & =[\log X],
\end{aligned}
$$

where $[\alpha]$ denotes the integer part of the real number $\alpha$;

$$
K=X^{15 / 14-c}(\log X)^{-6},
$$

$m, n, k, l, d, r$ (with or without subscripts) - integers,

$p, p_{1}, p_{2}, \ldots$ - prime numbers,

$\tau(n)$ - the number of positive divisors of $n$,

$\Lambda(n)$ - von Mangoldt's function,

$\Psi(x)=\sum_{n \leq x} \Lambda(n)$ - Chebyshev's function,

$\varrho=\beta+i \gamma$ - non-trivial zero of the Riemann zeta function $\zeta(s)$,

$\sum_{a<\gamma<b}$ - sum over the non-trivial zeroes of $\zeta(s)$ such that $a<\gamma<b$, $e(x)=e^{2 \pi i x}$,

$A \asymp B$ means $A \ll B \ll A$,

$$
\begin{aligned}
S(x) & =\sum_{X / 2<p \leq X} \log p \cdot e\left(p^{c} x\right), \\
I(x) & =\int_{X / 2}^{X} e\left(t^{c} x\right) d t, \\
I_{\varrho}(x) & =\int_{X / 2}^{X} e\left(t^{c} x\right) t^{\varrho-1} d t, \\
J(x) & =\sum_{|\gamma| \leq T} I_{\varrho}(x) .
\end{aligned}
$$

The constants in $O$-terms and $\ll$-symbols are absolute or depend only on $c$.

To prove the theorem we need some lemmas.

Lemma 1. Let $a, \delta$ be real numbers, $0<\delta<a / 4$, and let $k$ be an integer. There exists a function $\varphi(y)$ which is $k$ times continuously differentiable and such that

$$
\begin{aligned}
\varphi(y)=1 & \text { for }|y| \leq a-\delta, \\
0<\varphi(y)<1 & \text { for } a-\delta<|y|<a+\delta, \\
\varphi(y)=0 & \text { for }|y| \geq a+\delta,
\end{aligned}
$$


and its Fourier transform

$$
\Phi(x)=\int_{-\infty}^{\infty} e(-x y) \varphi(y) d y
$$

satisfies the inequality

$$
|\Phi(x)| \leq \min \left(2 a, \frac{1}{\pi|x|}, \frac{1}{\pi|x|}\left(\frac{k}{2 \pi|x| \delta}\right)^{k}\right) .
$$

Proof. See [4] or [5].

Throughout this paper we denote by $\varphi(y)$ the function from Lemma 1 with parameters $a=\frac{9}{10} \varepsilon, \delta=\Delta, k=r$, and by $\Phi(x)$ the Fourier transform of $\varphi(y)$.

Lemma 2. Assume that $G(x), F(x)$ are real functions defined in $[a, b]$, $|G(x)| \leq H$ for $a \leq x \leq b$ and $G(x) / F^{\prime}(x)$ is a monotonous function. Set

$$
I=\int_{a}^{b} G(x) e(F(x)) d x .
$$

If $F^{\prime}(x) \geq h>0$ for all $x \in[a, b]$ or if $F^{\prime}(x) \leq-h<0$ for all $x \in[a, b]$ then

$$
|I| \ll H / h .
$$

If $F^{\prime \prime}(x) \geq h>0$ for all $x \in[a, b]$ then

$$
|I| \ll H / \sqrt{h} \text {. }
$$

Proof. See [6, p. 71].

Lemma 3. Suppose that $f^{\prime \prime}(t)$ exists, is continuous on $[a, b]$ and satisfies

$$
f^{\prime \prime}(t) \asymp \lambda \quad(\lambda>0) \quad \text { for } t \in[a, b] .
$$

Then

$$
\left|\sum_{a<n \leq b} e(f(n))\right| \ll(b-a) \lambda^{1 / 2}+\lambda^{-1 / 2} .
$$

Proof. See [6, p. 104].

Lemma 4. If $2 \leq t \leq x$ then

$$
\Psi(x)=x-\sum_{|\gamma| \leq t} \frac{x^{\varrho}}{\varrho}+O\left(\frac{x \log ^{2} x}{t}\right) .
$$

Proof. See $[3$, p. 80$]$. 
Lemma 5. Assume that $1 \leq R \leq Y$. There exists a number $\gamma_{1}>0$ such that if

$$
\theta(R)=\frac{\gamma_{1}}{\log ^{2 / 3}(R+10) \log \log (R+10)}
$$

then the following estimate holds:

$$
\sum_{0<\gamma \leq R} Y^{\beta} \ll \begin{cases}Y^{1 / 2} R \log ^{6} Y, & Y^{3 / 4} \leq R \leq Y, \\ e^{2 \log Y+3 \log R-2 \sqrt{3 \log Y \log R}} \log ^{6} Y, & Y^{1 / 3} \leq R \leq Y^{3 / 4}, \\ Y^{1-\theta(R)} R^{2.4 \theta(R)} \log ^{45} Y, & 1 \leq R \leq Y^{1 / 3} .\end{cases}
$$

Proof. This lemma can be deduced from the density theorems of Ingham (see [6, p. 236]) and Huxley (see [2]) combined with the estimate of I. M. Vinogradov for the zero-free region of $\zeta(s)$ (see [3, p. 100]). Detailed computations may be found in [1].

LEMMA 6. We have

$$
\int_{-\infty}^{\infty} I^{3}(x) e(-N x) \Phi(x) d x \gg \varepsilon X^{3-c} .
$$

Proof. Denote the above integral by $H$. We have

$$
H=\int_{X / \mathbb{X} / \mathbb{X} / 2}^{X} \int_{-\infty}^{X} \int_{-\infty}^{X} e\left(\left(t_{1}^{c}+t_{2}^{c}+t_{3}^{c}-N\right) x\right) \Phi(x) d x d t_{1} d t_{2} d t_{3}
$$

(the change of the order of integration is legitimate because of the absolute convergence of the integral). Using the Fourier inversion formula we get

$$
H=\int_{X / \mathbb{X} / \mathbb{X} / 2}^{X} \int_{1}^{X} \varphi\left(t_{1}^{c}+t_{2}^{c}+t_{3}^{c}-N\right) d t_{1} d t_{2} d t_{3}
$$

and by the definition of $\varphi(y)$ we get

$$
\begin{aligned}
& H \geq \int_{X / \mathscr{X} / \mathbb{X} / 2}^{X} \int_{\substack{X \\
X}}^{X} d t_{1} d t_{2} d t_{3} \geq \int_{\lambda \chi X}^{\mu X \mu X} \int_{\mathfrak{M}}\left(\int_{\mathfrak{M}} d t_{3}\right) d t_{1} d t_{2}, \\
& \left|t_{1}^{c}+t_{2}^{c}+t_{3}^{c}-N\right|<4 \varepsilon / 5
\end{aligned}
$$

where $\lambda$ and $\mu$ are real numbers such that

$$
\frac{1}{2}<\left(\frac{1}{2}\right)^{1 / c}<\lambda<\mu<\left(\frac{1}{2}\left(2-\frac{1}{2^{c}}\right)\right)^{1 / c}<1
$$

and

$$
\mathfrak{M}=[X / 2, X] \cap\left[\left(N-\frac{4}{5} \varepsilon-t_{1}^{c}-t_{2}^{c}\right)^{1 / c},\left(N+\frac{4}{5} \varepsilon-t_{1}^{c}-t_{2}^{c}\right)^{1 / c}\right] .
$$

Because of (13) and the choice of $N, X, \varepsilon$ it is easy to show that in fact $\mathfrak{M}$ is the right interval of the above intersection. Thus by the mean-value 
theorem

$$
H \gg \varepsilon \int_{\lambda X}^{\mu X} \int_{\lambda X}^{\mu X}\left(\xi_{t_{1}, t_{2}}\right)^{1 / c-1} d t_{1} d t_{2},
$$

where $\xi_{t_{1}, t_{2}} \asymp X^{c}$. Therefore, $H \gg \varepsilon X^{3-c}$, which proves the lemma.

LEMMA 7. We have

$$
\begin{aligned}
& \int_{-\tau}^{\tau}\left|S^{2}(x)\right| d x \ll X^{2-c} \log ^{3} X, \\
& \int_{-\tau}^{\tau}\left|I^{2}(x)\right| d x \ll X^{2-c} \log X, \\
& \int_{n}^{n+1}\left|S^{2}(x)\right| d x \ll X \log ^{3} X
\end{aligned}
$$

uniformly with respect to $n$.

Proof. We only prove (i). Inequalities (ii) and (iii) can be proved likewise.

We have

$$
\begin{aligned}
\int_{-\tau}^{\tau}\left|S^{2}(x)\right| d x & =\sum_{X / 2<p_{1}, p_{2} \leq X} \log p_{1} \log p_{2} \int_{-\tau}^{\tau} e\left(\left(p_{1}^{c}-p_{2}^{c}\right) x\right) d x \\
& \ll \sum_{X / 2<p_{1}, p_{2} \leq X} \log p_{1} \log p_{2} \min \left(\tau, \frac{1}{\left|p_{1}^{c}-p_{2}^{c}\right|}\right) \\
& \ll U \tau \log ^{2} X+V \log ^{2} X
\end{aligned}
$$

where

$$
U=\sum_{\substack{X / 2<n_{1}, n_{2} \leq X \\\left|n_{1}^{c}-n_{2}^{c}\right| \leq 1 / \tau}} 1, \quad V=\sum_{\substack{X / 2<n_{1}, n_{2} \leq X \\ 1 / \tau<\left|n_{1}^{c}-n_{2}^{c}\right|}} \frac{1}{\left|n_{1}^{c}-n_{2}^{c}\right|} .
$$

We have

$$
U \ll \sum_{\substack{X / 2<n_{1} \leq X \\\left(n_{1}^{c}-1 / \tau\right)^{1 / c} \leq n_{2} \leq\left(n_{1}^{c}+1 / \tau\right)^{1 / c}}} \sum_{X / 2<n_{2} \leq X} 1 \ll \sum_{X / 2<n_{1} \leq X}\left(1+\left(n_{1}^{c}+1 / \tau\right)^{1 / c}-\left(n_{1}^{c}-1 / \tau\right)^{1 / c}\right)
$$

and by the mean-value theorem

$$
U \ll X+\frac{1}{\tau} X^{2-c} .
$$


Obviously $V \leq \sum_{l} V_{l}$ where

$$
V_{l}=\sum_{\substack{X / 2<n_{1}, n_{2} \leq X \\ l<\left|n_{1}^{c}-n_{2}^{c}\right| \leq 2 l}} \frac{1}{\left|n_{1}^{c}-n_{2}^{c}\right|}
$$

and $l$ takes the values $2^{k} / \tau, k=0,1,2, \ldots$, with $l \leq X^{c}$. We have

$$
V_{l} \ll \frac{1}{l} \sum_{\substack{X / 2<n_{1} \leq X \\\left(n_{1}^{c}+l\right)^{1 / c} \leq n_{2} \leq\left(n_{1}^{c}+2 l\right)^{1 / c}}} \sum_{X / 2<n_{2} \leq X} 1 .
$$

For $l \geq 1 / \tau$ and $X / 2<n_{1} \leq X$ it is easy to see that

$$
\left(n_{1}^{c}+2 l\right)^{1 / c}-\left(n_{1}^{c}+l\right)^{1 / c}>1 .
$$

Hence

$$
V_{l} \ll \frac{1}{l} \sum_{X / 2<n_{1} \leq X}\left(\left(n_{1}^{c}+2 l\right)^{1 / c}-\left(n_{1}^{c}+l\right)^{1 / c}\right) \ll X^{2-c}
$$

by the mean-value theorem.

The conclusion follows from formulas (3) and (14)-(17).

Lemma 8. Let $a_{m}, b_{n}$ be arbitrary complex numbers and let

$$
\begin{aligned}
& \tau \leq|x| \leq K, \quad X^{1 / 4}<R \leq X^{1 / 2}, \\
& X^{1 / 4}<L<L_{1} \leq 2 L, \quad L R \leq X .
\end{aligned}
$$

Define

$$
W=\sum_{L<n \leq L_{1}} \sum_{X^{1 / 4}<m \leq R} a_{m} b_{n} e\left((m n)^{c} x\right)
$$

Then

$$
|W| \ll(\mathcal{A B})^{1 / 2} X^{3 / 7}(\log X)^{-1},
$$

where

$$
\mathcal{A}=\sum_{X^{1 / 4}<m \leq R}\left|a_{m}\right|^{2}, \quad \mathcal{B}=\sum_{L<n \leq L_{1}}\left|b_{n}\right|^{2} .
$$

Proof. Obviously, we may suppose that $\tau \leq x \leq K$. Take $s \in[1, R]$ whose exact value will be determined later. We define the numbers $R_{i}$, $0 \leq i \leq Q$, in the following way:

$$
R_{0}=X^{1 / 4}, \quad R_{i+1}=\min \left(R_{i}+s, R\right), \quad R_{Q}=R .
$$

Obviously $Q \ll R / s$. We have

$$
W=\sum_{L<n \leq L_{1}} \sum_{1 \leq i \leq Q} \sum_{R_{i-1}<m \leq R_{i}} a_{m} b_{n} e\left((m n)^{c} x\right),
$$


hence

$$
|W| \leq \sum_{L<n \leq L_{1}}\left|b_{n}\right|\left|\sum_{1 \leq i \leq Q} \sum_{R_{i-1}<m \leq R_{i}} a_{m} e\left((m n)^{c} x\right)\right|
$$

and Cauchy's inequality gives

$$
\begin{aligned}
|W|^{2} & \leq \mathcal{B} \sum_{L<n \leq L_{1}}\left|\sum_{1 \leq i \leq Q} \sum_{R_{i-1}<m \leq R_{i}} a_{m} e\left((m n)^{c} x\right)\right|^{2} \\
& \leq \mathcal{B} Q \sum_{L<n \leq L_{1}} \sum_{1 \leq i \leq Q}\left|\sum_{R_{i-1}<m \leq R_{i}} a_{m} e\left((m n)^{c} x\right)\right|^{2} \\
& =\mathcal{B} Q \sum_{L<n \leq L_{1}} \sum_{1 \leq i \leq Q} \sum_{R_{i-1}<m_{1}, m_{2} \leq R_{i}} a_{m_{1}} \bar{a}_{m_{2}} e\left(\left(m_{1}^{c}-m_{2}^{c}\right) n^{c} x\right) .
\end{aligned}
$$

After some rearrangements we obtain

$$
\begin{aligned}
&|W|^{2} \ll \mathcal{B} Q\left(\sum_{L<n \leq L_{1}} \sum_{1 \leq i \leq Q} \sum_{R_{i-1}<m \leq R_{i}}\left|a_{m}\right|^{2}\right. \\
&+\sum_{1 \leq i \leq Q} \sum_{\substack{R_{i-1}<m_{1}, m_{2} \leq R_{i} \\
m_{1} \neq m_{2}}}\left|a_{m_{1}}\right|\left|a_{m_{2}}\right| \\
&\left.\times\left|\sum_{L<n \leq L_{1}} e\left(\left(m_{1}^{c}-m_{2}^{c}\right) n^{c} x\right)\right|\right) \\
& \sum_{1 \leq h \leq s} \mid \mathcal{B} Q\left(\mathcal{A} L+\sum_{1 \leq i \leq Q} \sum_{R_{i-1}<m \leq R_{i}-h}|| a_{m+h} \mid\right. \\
&\left.\times\left|\sum_{L<n \leq L_{1}} e(f(n))\right|\right),
\end{aligned}
$$

where

$$
f(n)=\left((m+h)^{c}-m^{c}\right) n^{c} x .
$$

It is easy to see that if $L<n \leq L_{1}$ then

$$
f^{\prime \prime}(n) \asymp\left((m+h)^{c}-m^{c}\right) L^{c-2} x
$$

and by Lemma 3 we obtain

$$
\begin{aligned}
\left|\sum_{L<n \leq L_{1}} e(f(n))\right| \ll & \left((m+h)^{c}-m^{c}\right)^{1 / 2} L^{c / 2} x^{1 / 2} \\
& +\left((m+h)^{c}-m^{c}\right)^{-1 / 2} L^{1-c / 2} x^{-1 / 2} \\
\ll & \left((m+h)^{c}-m^{c}\right)^{1 / 2} L^{c / 2} K^{1 / 2} \\
& +\left((m+h)^{c}-m^{c}\right)^{-1 / 2} L^{1-c / 2} \tau^{-1 / 2} \\
\ll & \left((m+h)^{c}-m^{c}\right)^{1 / 2} L^{c / 2} K^{1 / 2} \\
\ll & s^{1 / 2} R^{(c-1) / 2} L^{c / 2} K^{1 / 2} .
\end{aligned}
$$


We substitute this estimate in (18) to get

$$
|W|^{2} \ll \mathcal{B} Q\left(\mathcal{A} L+s^{1 / 2} R^{(c-1) / 2} L^{c / 2} K^{1 / 2} \Sigma_{0}\right),
$$

where

$$
\Sigma_{0}=\sum_{1 \leq h \leq s} \sum_{1 \leq i \leq Q} \sum_{R_{i-1}<m \leq R_{i}-h}\left|a_{m}\right|\left|a_{m+h}\right| .
$$

Clearly

$$
\begin{aligned}
\Sigma_{0} & \leq \sum_{1 \leq h \leq s} \sum_{X^{1 / 4}<m \leq R-h}\left|a_{m}\right|\left|a_{m+h}\right| \\
& \leq \sum_{1 \leq h \leq s}\left(\sum_{X^{1 / 4}<m \leq R-h}\left|a_{m}\right|^{2}\right)^{1 / 2}\left(\sum_{X^{1 / 4}<m \leq R-h}\left|a_{m+h}\right|^{2}\right)^{1 / 2} \leq s \mathcal{A} .
\end{aligned}
$$

The last estimate, (19) and the inequality $Q \ll R / s$ give us

$$
|W|^{2} \ll \mathcal{A B} L R\left(1 / s+s^{1 / 2} R^{(c-1) / 2} L^{(c-2) / 2} K^{1 / 2}\right) .
$$

We now determine $s$ in such a way that the two summands in brackets are equal. This gives the value

$$
s=K^{-1 / 3} R^{(1-c) / 3} L^{(2-c) / 3} .
$$

The assumption of our lemma and (8) imply easily $1 \leq s \leq R$. Substituting $s$ in (20) we get after some calculations

$$
|W|^{2} \ll \mathcal{A B} X^{6 / 7}(\log X)^{-2},
$$

i.e.

$$
|W| \ll(\mathcal{A B})^{1 / 2} X^{3 / 7}(\log X)^{-1},
$$

which is the desired result.

Lemma 9. Suppose that $a_{m}, b_{n}$ are arbitrary complex numbers and that $L<L_{1} \leq 2 L, L \leq X$. Set

$$
V=\sum_{L<n \leq L_{1}} \sum_{X^{1 / 4}<m \leq X / n} a_{m} b_{n} e\left((m n)^{c} x\right) .
$$

Then

$$
|V| \ll \log X \cdot\left|\sum_{L<n \leq L_{1}} \sum_{X^{1 / 4}<m \leq X / L} a_{m}^{\prime} b_{n}^{\prime} e\left((m n)^{c} x\right)\right|
$$

for some complex numbers $a_{m}^{\prime}, b_{n}^{\prime}$ such that $\left|a_{m}^{\prime}\right| \leq\left|a_{m}\right|,\left|b_{n}^{\prime}\right| \leq\left|b_{n}\right|$.

Proof. Let $q$ be an odd integer such that $2 X / L \leq q \leq 4 X / L$. We have

(21) $V=\sum_{L<n \leq L_{1}} \sum_{X^{1 / 4}<m \leq X / L} a_{m} b_{n} e\left((m n)^{c} x\right)$

$$
\times \sum_{X^{1 / 4}<m_{1} \leq X / n} \frac{1}{q} \sum_{|k| \leq(q-1) / 2} e\left(\frac{k\left(m-m_{1}\right)}{q}\right)
$$




$$
=\sum_{|k| \leq(q-1) / 2} \frac{1}{\max (1,|k|)} \sum_{L<n \leq L_{1}} \sum_{X^{1 / 4}<m \leq X / L} a_{m}^{(k)} b_{n}^{(k)} e\left((m n)^{c} x\right),
$$

where

$$
a_{m}^{(k)}=a_{m} e\left(\frac{k m}{q}\right), \quad b_{n}^{(k)}=b_{n} \frac{\max (1,|k|)}{q} \sum_{X^{1 / 4}<m_{1} \leq X / n} e\left(-\frac{k m_{1}}{q}\right) .
$$

Obviously $\left|a_{m}^{(k)}\right|=\left|a_{m}\right|$ and because of the well-known estimate

$$
\left|\sum_{a<k \leq b} e(\alpha k)\right| \ll \min (b-a, 1 /\|\alpha\|)
$$

we get

$$
\left|b_{n}^{(k)}\right| \ll\left|b_{n}\right| .
$$

The absolute value of the double sum over $m$ and $n$ on the right-hand side of (21) takes its maximum for some value $k=k_{0}$.

Denote by $a_{m}^{\prime}, b_{n}^{\prime}$ the numbers $a_{m}^{\left(k_{0}\right)}, b_{n}^{\left(k_{0}\right)}$, multiplied by a sufficiently small positive constant.

Obviously this proves the lemma.

Lemma 10. Assume that $\tau \leq|x| \leq K$. Then

$$
|S(x)| \ll X^{13 / 14} \log ^{3} X .
$$

Proof. Without loss of generality we may assume that $\tau \leq x \leq K$. Clearly

$$
S(x)=V_{0}(x)-V_{1}(x)+O\left(X^{1 / 2}\right)
$$

where

$$
V_{i}(x)=\sum_{X^{1 / 4}<n \leq 2^{-i} X} \Lambda(n) e\left(n^{c} x\right), \quad i=0,1 .
$$

Hence it is sufficient to prove that

$$
\left|V_{0}(x)\right|,\left|V_{1}(x)\right| \ll X^{13 / 14} \log ^{3} X .
$$

Consider, for instance, $V_{0}(x)$. We use Vaughan's identity (see [8]) to get

$$
V_{0}(x)=S_{1}-S_{2}-S_{3},
$$

where

$$
\begin{aligned}
S_{1} & =\sum_{d \leq X^{1 / 4}} \mu(d) \sum_{l \leq X / d} \log l \cdot e\left((l d)^{c} x\right), \\
S_{2} & =\sum_{k \leq X^{1 / 2}} \sum_{r \leq X / k} c_{k} e\left((k r)^{c} x\right), \\
S_{3} & =\sum_{X^{1 / 4}<m \leq X^{3 / 4}} \sum_{X^{1 / 4}<n \leq X / m} a_{m} \Lambda(n) e\left((m n)^{c} x\right),
\end{aligned}
$$

where $\left|c_{k}\right| \leq \log k,\left|a_{m}\right| \leq \tau(m)$. 
We split the sum $S_{2}$ in the following way:

$$
S_{2}=S_{2}^{(1)}+S_{2}^{(2)}+O\left(X^{3 / 4} \log X\right),
$$

where

$$
\begin{aligned}
S_{2}^{(1)} & =\sum_{k \leq X^{1 / 4}} \sum_{r \leq X / k} c_{k} e\left((k r)^{c} x\right), \\
S_{2}^{(2)} & =\sum_{X^{1 / 4}<k \leq X^{1 / 2}} \sum_{X^{1 / 4}<r \leq X / k} c_{k} e\left((k r)^{c} x\right) .
\end{aligned}
$$

Let us treat $S_{1}$. We have

$$
\left|S_{1}\right| \leq \sum_{d \leq X^{1 / 4}}\left|\sum_{l \leq X / d} \log l \cdot e\left((l d)^{c} x\right)\right| .
$$

We break the sum over $l$ into sums of the type

$$
T_{L}=\sum_{L<l \leq L_{1}} \log l \cdot e\left((l d)^{c} x\right)
$$

whose number is $O(\log X)$ and $L<L_{1} \leq 2 L, L_{1} \leq X / d$. Abel's transformation formula gives us

$$
\left|T_{L}\right| \ll \log X \max _{L_{2} \in\left[L, L_{1}\right]}\left|\sum_{L<l \leq L_{2}} e(g(l))\right|,
$$

where $g(l)=(l d)^{c} x$. Obviously $g^{\prime \prime}(l) \asymp L^{c-2} d^{c} x$ for $L \leq l \leq L_{2}$ and by Lemma 3

$$
\begin{aligned}
\left|\sum_{L<l \leq L_{2}} e(g(l))\right| & \ll L^{c / 2} d^{c / 2} x^{1 / 2}+L^{1-c / 2} d^{-c / 2} x^{-1 / 2} \\
& \leq L^{c / 2} d^{c / 2} K^{1 / 2}+L^{1-c / 2} d^{-c / 2} \tau^{-1 / 2} \\
& \leq X^{c / 2} K^{1 / 2}+X^{1-c / 2} d^{-1} \tau^{-1 / 2} \ll X^{c / 2} K^{1 / 2} .
\end{aligned}
$$

The last estimate, together with (25) and (26), implies

$$
\left|S_{1}\right| \ll \log ^{2} X \cdot X^{1 / 4+c / 2} K^{1 / 2} \ll X^{11 / 14} .
$$

We estimate $S_{2}^{(1)}$ in the same way to get

$$
\left|S_{2}^{(1)}\right| \ll X^{11 / 14} \text {. }
$$

To estimate $S_{2}^{(2)}$ and $S_{3}$ we need Lemmas 8 and 9 . Consider $S_{3}$. We split it in the following way:

$$
S_{3}=W_{1}+W_{2}+W_{3},
$$


where

$$
\begin{aligned}
& W_{1}=\sum_{X^{1 / 2}<n \leq X^{3 / 4}} \sum_{X^{1 / 4}<m \leq X / n} a_{m} \Lambda(n) e\left((m n)^{c} x\right), \\
& W_{2}=\sum_{X^{1 / 2}<n \leq X^{3 / 4}} \sum_{X^{1 / 4}<m \leq X / n} \Lambda(m) a_{n} e\left((m n)^{c} x\right), \\
& W_{3}=\sum_{X^{1 / 4}<n \leq X^{1 / 2}} \sum_{X^{1 / 4}<m \leq X^{1 / 2}} a_{m} \Lambda(n) e\left((m n)^{c} x\right) .
\end{aligned}
$$

We break $W_{1}$ into sums of the type

$$
W_{1}(L)=\sum_{L<n \leq L_{1}} \sum_{X^{1 / 4}<m \leq X / n} a_{m} \Lambda(n) e\left((m n)^{c} x\right)
$$

whose number is $O(\log X)$ and $X^{1 / 2} \leq L<L_{1} \leq X^{3 / 4}, L_{1} \leq 2 L$. According to Lemma 9 there exist complex numbers $a_{m}^{\prime}, b_{n}^{\prime}$ such that $\left|a_{m}^{\prime}\right| \leq\left|a_{m}\right| \leq$ $\tau(m),\left|b_{n}^{\prime}\right| \leq \Lambda(n)$ and

$$
\left|W_{1}(L)\right| \ll(\log X) \cdot\left|W_{1}^{\prime}(L)\right|,
$$

where

$$
W_{1}^{\prime}(L)=\sum_{L<n \leq L_{1}} \sum_{X^{1 / 4}<m \leq X / L} a_{m}^{\prime} b_{n}^{\prime} e\left((m n)^{c} x\right) .
$$

Using the well-known inequalities

$$
\sum_{m \leq y} \tau^{2}(m) \ll y \log ^{3} y, \quad \sum_{n \leq y} \Lambda^{2}(n) \ll y \log y
$$

and Lemma 8 we obtain

$$
\left|W_{1}^{\prime}(L)\right| \ll\left(\frac{X}{L} \log ^{3} X \cdot L \log X\right)^{1 / 2} X^{3 / 7}(\log X)^{-1}=X^{13 / 14} \log X,
$$

hence

$$
\left|W_{1}\right| \ll X^{13 / 14} \log ^{3} X .
$$

We estimate $W_{2}$ and $W_{3}$ in the same way (for $W_{3}$, of course, we do not use Lemma 9) to get

$$
\left|W_{2}\right|,\left|W_{3}\right| \ll X^{13 / 14} \log ^{3} X
$$

We treat $S_{2}^{(2)}$ analogously:

$$
S_{2}^{(2)}=U_{1}+U_{2},
$$


where

$$
\begin{aligned}
U_{1} & =\sum_{X^{1 / 4}<r \leq X^{1 / 2}} \sum_{X^{1 / 4}<k \leq X^{1 / 2}} c_{k} e\left((k r)^{c} x\right), \\
U_{2} & =\sum_{X^{1 / 2}<r \leq X^{3 / 4}} \sum_{X^{1 / 4}<k \leq X / r} c_{k} e\left((k r)^{c} x\right) .
\end{aligned}
$$

We estimate these sums just as $W_{1}, W_{2}, W_{3}$, with the help of Lemmas 8 and 9 , using the inequality

$$
\sum_{k \leq y} \log ^{2} k \ll y \log ^{2} y .
$$

Thus we get

$$
\left|U_{1}\right|,\left|U_{2}\right| \ll X^{13 / 14} \log ^{3} X
$$

The inequality (22) follows from (23), (24), (27)-(33). The lemma is proved.

LEMma 11. If $1 \leq T_{1} \leq T$ then

$$
\frac{1}{\sqrt{T_{1}}} \sum_{0<\gamma \leq T_{1}} X^{\beta} \ll X e^{-(\log X)^{1 / 4}} .
$$

Proof. Assume that $T_{1} \leq X^{1 / 3}$. Then using Lemma 5 we have

$$
\begin{aligned}
\frac{1}{\sqrt{T_{1}}} \sum_{0<\gamma \leq T_{1}} X^{\beta} & \leq \sum_{0<\gamma \leq T_{1}} X^{\beta} \leq \sum_{0<\gamma \leq X^{1 / 3}} X^{\beta} \\
& \ll \log ^{45} X \cdot X^{1-0.2 \theta\left(X^{1 / 3}\right)} \ll X e^{-(\log X)^{1 / 4}} .
\end{aligned}
$$

If $X^{1 / 3} \leq T_{1} \leq X^{3 / 4}$ then using Lemma 5 we get

$$
\frac{1}{\sqrt{T_{1}}} \sum_{0<\gamma \leq T_{1}} X^{\beta} \ll \log ^{6} X \cdot e^{\omega}
$$

where

$$
\omega=2 \log X+\frac{5}{2} \log T_{1}-2 \sqrt{3 \log X \log T_{1}} .
$$

Obviously $\omega$ is a convex function of $\sqrt{\log T_{1}}$. Thus

$$
\max _{X^{1 / 3} \leq T_{1} \leq X^{3 / 4}} \omega=\max \left(\left.\omega\right|_{T_{1}=X^{1 / 3}},\left.\omega\right|_{T_{1}=X^{3 / 4}}\right)=\frac{7}{8} \log X .
$$

Hence

$$
\log ^{6} X \cdot e^{\omega} \ll X^{7 / 8} \log ^{6} X \ll X e^{-(\log X)^{1 / 4}} .
$$

If $X^{3 / 4} \leq T_{1} \leq T$ then Lemma 5 gives us

$$
\begin{aligned}
\frac{1}{\sqrt{T_{1}}} \sum_{0<\gamma \leq T_{1}} X^{\beta} & \ll \log ^{6} X \cdot \frac{1}{\sqrt{T_{1}}} X^{1 / 2} T_{1} \\
& \leq \log ^{6} X \cdot X^{1 / 2} T^{1 / 2} \ll X e^{-(\log X)^{1 / 4}} .
\end{aligned}
$$


Lemma 11 is proved.

Lemma 12. If $1 \leq T_{1} \leq T$ then

$$
\frac{1}{T_{1}} \sum_{0<\gamma \leq T_{1}} X^{\beta} \ll X e^{-(\log X)^{1 / 4}} .
$$

P r o of. This lemma follows immediately from Lemma 11.

LEMma 13. If $|x| \leq \tau$ then

$$
|J(x)| \ll X e^{-(\log X)^{1 / 5}},
$$

where $J(x)$ is defined in (12).

Proof. Without loss of generality we may assume that $x \geq 0$. By the definition we have

$$
I_{\varrho}(x)=\int_{X / 2}^{X} t^{\beta-1} e(F(t)) d t
$$

where

$$
F(t)=t^{c} x+\frac{\gamma}{2 \pi} \log t .
$$

Define the following sets of non-trivial zeroes of $\zeta(s)$ :

$$
\begin{aligned}
& M_{1}=\left\{\varrho|| \gamma \mid \leq T,-\gamma / 2 \pi>\frac{3}{2} c X^{c} x\right\}, \\
& M_{2}=\left\{\varrho|| \gamma \mid \leq T, \frac{1}{2} c(X / 2)^{c} x \leq-\gamma / 2 \pi \leq \frac{3}{2} c X^{c} x\right\}, \\
& M_{3}=\left\{\varrho|| \gamma \mid \leq T,-\gamma / 2 \pi<\frac{1}{2} c(X / 2)^{c} x\right\} .
\end{aligned}
$$

(The set $M_{2}$ may be empty.)

Let $X^{-c} \leq x \leq \tau$. If $\varrho \in M_{3}$ then

$$
F^{\prime}(t) \gg \frac{1}{X}\left(c\left(\frac{X}{2}\right)^{c} x-\frac{-\gamma}{2 \pi}\right)>0
$$

and according to Lemma 2

$$
\left|I_{\varrho}(x)\right| \ll \frac{X^{\beta}}{c(X / 2)^{c} x+\gamma / 2 \pi} .
$$

Hence

$$
\begin{aligned}
\sum_{\varrho \in M_{3}}\left|I_{\varrho}(x)\right| & \ll \sum_{-\pi c(X / 2)^{c} x \leq \gamma \leq T} \frac{X^{\beta}}{c(X / 2)^{c} x+\gamma / 2 \pi} \\
& \ll \sum_{-\pi c(X / 2)^{c} x \leq \gamma \leq X^{c} x} \frac{X^{\beta}}{X^{c} x}+\sum_{X^{c} x<\gamma \leq T} \frac{X^{\beta}}{\gamma} \\
& \ll \log X \max _{1 \leq T_{1} \leq T}\left(\frac{1}{T_{1}} \sum_{0<\gamma \leq T_{1}} X^{\beta}\right) .
\end{aligned}
$$


Using Lemma 12 we obtain

$$
\sum_{\varrho \in M_{3}}\left|I_{\varrho}(x)\right| \ll X e^{-(\log X)^{1 / 5}} .
$$

If $\varrho \in M_{1}$ then

$$
F^{\prime}(t) \leq \frac{1}{t}\left(c X^{c} x+\frac{\gamma}{2 \pi}\right) \leq \frac{1}{X}\left(c X^{c} x+\frac{\gamma}{2 \pi}\right)<0
$$

and by Lemma 2 we get

Therefore

$$
\left|I_{\varrho}(x)\right| \ll \frac{X^{\beta}}{-\gamma / 2 \pi-c X^{c} x} \ll \frac{X^{\beta}}{|\gamma|} .
$$

$$
\begin{aligned}
\sum_{\varrho \in M_{1}}\left|I_{\varrho}(x)\right| & \ll \sum_{0<\gamma \leq T_{1}} \frac{X^{\beta}}{\gamma} \\
& \ll \log X \cdot \max _{1 \leq T_{1} \leq T}\left(\frac{1}{T_{1}} \sum_{0<\gamma \leq T_{1}} X^{\beta}\right)
\end{aligned}
$$

and Lemma 12 gives

$$
\sum_{\varrho \in M_{1}}\left|I_{\varrho}(x)\right| \ll X e^{-(\log X)^{1 / 5}} .
$$

If $\varrho \in M_{2}$ then $-\gamma / 2 \pi \asymp X^{c} x$ and $F^{\prime \prime}(t) \asymp X^{c-2} x$. According to Lemma 2

Hence, using Lemma 11 we get

$$
\left|I_{\varrho}(x)\right| \ll \frac{X^{\beta}}{\sqrt{X^{c} x}} .
$$

$$
\sum_{\varrho \in M_{2}}\left|I_{\varrho}(x)\right| \ll \frac{1}{\sqrt{X^{c} x}} \sum_{0<\gamma \leq X^{c} x} X^{\beta} \ll X e^{-(\log X)^{1 / 4}} .
$$

(If the set $M_{2}$ is empty then the last estimate is also true.)

Inequalities (34)-(36) give us

$$
|J(x)| \ll X e^{-(\log X)^{1 / 5}} .
$$

If $0 \leq x \leq X^{-c}$ then estimating $\left|I_{\varrho}(x)\right|$ trivially we have

$$
\sum_{\varrho \in M_{2}}\left|I_{\varrho}(x)\right| \ll \sum_{\varrho \in M_{2}} X^{\beta} \ll X^{\beta_{0}},
$$

where $\beta_{0}=\max _{\varrho \in M_{2}} \beta<1$. The terms $\sum_{\varrho \in M_{1}}\left|I_{\varrho}(x)\right|$ and $\sum_{\varrho \in M_{3}}\left|I_{\varrho}(x)\right|$ can be estimated as in the previous case. Thus (37) is always true.

Lemma 14. If $|x| \leq \tau$ then

$$
S(x)=I(x)+O\left(X e^{-(\log X)^{1 / 5}}\right) .
$$


Proof. Obviously

$$
S(x)=U(x)+O\left(X^{1 / 2}\right),
$$

where

$$
U(x)=\sum_{X / 2<n \leq X} \Lambda(n) e\left(n^{c} x\right) .
$$

Using Abel's transformation we get

$$
\begin{aligned}
U(x)= & -\int_{X / 2}^{X}(\Psi(t)-\Psi(X / 2)) \frac{d}{d t}\left(e\left(t^{c} x\right)\right) d t \\
& +(\Psi(X)-\Psi(X / 2)) e\left(X^{c} x\right) .
\end{aligned}
$$

According to Lemma 4 we obtain

$$
\begin{aligned}
U(x)= & -\int_{X / 2}^{X}\left(t-\frac{X}{2}-\sum_{|\gamma| \leq T} \frac{t^{\varrho}-(X / 2)^{\varrho}}{\varrho}\right. \\
& \left.+O\left(\frac{X \log ^{2} X}{T}\right)\right) \frac{d}{d t}\left(e\left(t^{c} x\right)\right) d t \\
& +\left(X-\frac{X}{2}-\sum_{|\gamma| \leq T} \frac{X^{\varrho}-(X / 2)^{\varrho}}{\varrho}+O\left(\frac{X \log ^{2} X}{T}\right)\right) e\left(X^{c} x\right) \\
= & -\int_{X / 2}^{X}\left(t-\frac{X}{2}-\sum_{|\gamma| \leq T} \frac{t^{\varrho}-(X / 2)^{\varrho}}{\varrho}\right) \frac{d}{d t}\left(e\left(t^{c} x\right)\right) d t \\
& +\left(X-\frac{X}{2}-\sum_{|\gamma| \leq T} \frac{X^{\varrho}-(X / 2)^{\varrho}}{\varrho}\right) e\left(X^{c} x\right)+O\left(\frac{\tau X^{1+c}}{T} \log ^{2} X\right) .
\end{aligned}
$$

Using partial integration we get

$$
\begin{aligned}
U(x) & =\int_{X / 2}^{X} e\left(t^{c} x\right)\left(1-\sum_{|\gamma| \leq T} t^{\varrho-1}\right) d t+O\left(\frac{\tau X^{1+c}}{T} \log ^{2} X\right) \\
& =I(x)-J(x)+O\left(\frac{\tau X^{1+c}}{T} \log ^{2} X\right) .
\end{aligned}
$$

The conclusion follows from formulas (3), (4), (38) and Lemma 13.

Proof of the Theorem. It is sufficient to show that the sum

$$
B=\sum_{\substack{X / 2<p_{1}, p_{2}, p_{3} \leq X \\\left|p_{1}^{c}+p_{2}^{c}+p_{3}^{c}-N\right|<\varepsilon}} \log p_{1} \log p_{2} \log p_{3}
$$


tends to infinity as $X$ tends to infinity. Set

$$
B_{1}=\sum_{X / 2<p_{1}, p_{2}, p_{3} \leq X} \log p_{1} \log p_{2} \log p_{3} \cdot \varphi\left(p_{1}^{c}+p_{2}^{c}+p_{3}^{c}-N\right) .
$$

By the definition of $\varphi(y)$ we have

$$
B \geq B_{1} .
$$

The Fourier transformation formula gives us

$$
\begin{aligned}
B_{1} & =\sum_{X / 2<p_{1}, p_{2}, p_{3} \leq X} \log p_{1} \log p_{2} \log p_{3} \int_{-\infty}^{\infty} e\left(\left(p_{1}^{c}+p_{2}^{c}+p_{3}^{c}-N\right) x\right) \Phi(x) d x \\
& =\int_{-\infty}^{\infty} S^{3}(x) e(-N x) \Phi(x) d x .
\end{aligned}
$$

Let us represent $B_{1}$ in the form

$$
B_{1}=D_{1}+D_{2}+D_{3},
$$

where

$$
\begin{aligned}
& D_{1}=\int_{-\tau}^{\tau} S^{3}(x) e(-N x) \Phi(x) d x, \\
& D_{2}=\int_{\tau<|x|<K} S^{3}(x) e(-N x) \Phi(x) d x, \\
& D_{3}=\int_{|x|>K} S^{3}(x) e(-N x) \Phi(x) d x .
\end{aligned}
$$

Using (5)-(8) and Lemma 1 we have

$$
\begin{aligned}
\left|D_{3}\right| & \ll \int_{K}^{\infty}\left|S^{3}(x)\right||\Phi(x)| d x \\
& \ll X^{3} \int_{K}^{\infty} \frac{1}{x}\left(\frac{r}{2 \pi \Delta x}\right)^{r} d x \ll X^{3}\left(\frac{r}{2 \pi \Delta K}\right)^{r} \ll 1 .
\end{aligned}
$$

We next treat $D_{2}$ :

$$
\begin{aligned}
\left|D_{2}\right| & \ll \int_{\tau}^{K}\left|S^{3}(x)\right||\Phi(x)| d x \\
& \ll\left(\max _{\tau \leq x \leq K}|S(x)|\right) \int_{\tau}^{K}\left|S^{2}(x)\right||\Phi(x)| d x .
\end{aligned}
$$


According to Lemma 1 we get

$$
\begin{aligned}
\int_{\tau}^{K}\left|S^{2}(x)\right||\Phi(x)| d x \ll \varepsilon \int_{\tau}^{1 / \varepsilon}\left|S^{2}(x)\right| d x+\int_{1 / \varepsilon}^{K}\left|S^{2}(x)\right| \frac{d x}{x} \\
\ll \varepsilon \sum_{0 \leq n \leq 1 / \varepsilon} \int_{n}^{n+1}\left|S^{2}(x)\right| d x+\sum_{1 / \varepsilon-1 \leq n \leq K} \frac{1}{n} \int_{n}^{n+1}\left|S^{2}(x)\right| d x .
\end{aligned}
$$

The last estimate and Lemma 7(iii) give

$$
\int_{\tau}^{K}\left|S^{2}(x)\right||\Phi(x)| d x \ll X \log ^{4} X .
$$

Therefore, by (42) and by Lemma 10 we have

$$
\left|D_{2}\right| \ll \varepsilon \frac{X^{3-c}}{\log X} .
$$

We now find an asymptotic formula for $D_{1}$. Set

$$
H_{1}=\int_{-\tau}^{\tau} I^{3}(x) e(-N x) \Phi(x) d x .
$$

Using Lemmas 7(i), 7(ii) and 14 we have

$$
\begin{aligned}
\left|D_{1}-H_{1}\right| & \ll \int_{-\tau}^{\tau}\left|S^{3}(x)-I^{3}(x)\right||\Phi(x)| d x \\
& \ll \varepsilon \int_{-\tau}^{\tau}|S(x)-I(x)|\left(\left|S^{2}(x)\right|+\left|I^{2}(x)\right|\right) d x \\
& \ll \varepsilon X e^{-(\log X)^{1 / 5}}\left(\int_{-\tau}^{\tau}\left|S^{2}(x)\right| d x+\int_{-\tau}^{\tau}\left|I^{2}(x)\right| d x\right) \\
& \ll \varepsilon X^{3-c} e^{-(\log X)^{1 / 6}} .
\end{aligned}
$$

Define

$$
H=\int_{-\infty}^{\infty} I^{3}(x) e(-N x) \Phi(x) d x
$$

Then we have

$$
\left|H-H_{1}\right| \ll \int_{\tau}^{\infty}\left|I^{3}(x)\right||\Phi(x)| d x .
$$

By Lemma 2 we get $|I(x)| \ll 1 /\left(|x| X^{c-1}\right)$. Therefore, by (45) and Lemma 1 
we obtain

$$
\left|H-H_{1}\right| \ll \frac{1}{X^{3(c-1)}} \int_{\tau}^{\infty}|\Phi(x)| \frac{d x}{x^{3}} \ll \frac{\varepsilon}{X^{3(c-1)} \tau^{2}} \ll \varepsilon \frac{X^{3-c}}{\log X} .
$$

It now follows from formulas (40), (41), (43)-(46) that

$$
B_{1}=H+O\left(\frac{\varepsilon X^{3-c}}{\log X}\right) \text {. }
$$

Hence by Lemma 6 we have $B_{1} \gg \varepsilon X^{3-c}$. Together with (40) this implies that $B \gg \varepsilon X^{3-c}$, and so $B \rightarrow \infty$ as $X \rightarrow \infty$.

The Theorem is proved.

Finally, I would like to thank Professor A. A. Karatsuba for useful and encouraging discussions.

\section{References}

[1] S. A. Gritsenko, On a problem of I. M. Vinogradov, Mat. Zametki 39 (1987), 625-640 (in Russian).

[2] M. N. Huxley, On the difference between consecutive primes, Invent. Math. 15 (1972), 164-170.

[3] A. A. Karatsuba, Principles of Analytic Number Theory, Nauka, Moscow 1983 (in Russian).

[4] I. I. Piatetski-Shapiro, On a variant of Waring-Goldbach's problem, Mat. Sb. 30 (72) (1) (1952), 105-120 (in Russian).

[5] B. I. Segal, On a theorem analogous to Waring's theorem, Dokl. Akad. Nauk SSSR (N. S.) 2 (1933), 47-49 (in Russian).

[6] E. G. Titchmarsh, The Theory of the Riemann Zeta-function (revised by D. R. Heath-Brown), Clarendon Press, Oxford 1986.

[7] D. I. Tolev, Diophantine inequalities involving prime numbers, Thesis, Moscow University, 1990 (in Russian).

[8] R. C. Vaughan, On the distribution of ap modulo 1, Mathematika 24 (1977), 135141.

DEPARTMENT OF MATHEMATICS

UNIVERSITY OF PLOVDIV

"TSAR ASEN" 24

PLOVDIV 4000, BULGARIA 